Article

\title{
On $B V$-Algebras
}

In Ho Hwang ${ }^{1}$, Yong Lin Liu ${ }^{2}$ and Hee Sik Kim ${ }^{3, *}$

1 Department of Mathematics, Incheon National University, Incheon 22012, Korea; ho818@inu.ac.kr

2 School of Mathematics and Finance, Putian University, Putian 351100, China; ylliu@ptu.edu.cn

3 Department of Mathematics, Research Institute of Natural Sciences, Hanyang University, Seoul 04763, Korea

* Correspondences: heekim@hanyang.ac.kr; Tel.: +82-10-9276-5630 or +82-2-2220-0899

Received: 9 September 2020; Accepted: 12 October 2020; Published: 14 October 2020

\begin{abstract}
In this paper, we introduce the notion of a $B V$-algebra, and we show that a $B V$-algebra is logically equivalent to several algebras, i.e., $B M$-algebras, $B T$-algebras, $B O$-algebras and 0 -commutative $B$-algebras. Moreover, we show that a $B V$-algebra with $(F)$ is logically equivalent to several algebras, and we show some relationships between a $B V$-algebra with $(F)$ and several related algebras.
\end{abstract}

Keywords: $B V$-algebra; $B$-algebra; $B F$-algebra; $B M$-algebra; $B N$-algebra; $B O$-algebra; $B T$-algebra

MSC: 06F35; 20N02

\section{Introduction}

The notion of $B C K$-algebras was formulated by Iséki. The motivation of this notion is based on both set theory and propositional calculus (see [1]). As a generalization of this notion, $B C I_{-}, \mathrm{BCH}-$, $B C H-, B C C$-algebras have been developed by many researchers (see [2-4]). Jun et al. [5] introduced the notion of a $B H$-algebra, which is a generalization of $B C K / B C I / B$-algebras. The concept of $B$-algebras was introduced by Neggers and Kim [6]. Kim and Kim [7] defined the notion of a BM-algebra. They showed that a $B M$-algebra is equivalent to a 0 -commutative $B$-algebra. Kim and Kim [8] introduced the notion of a $B O$-algebra, and proved that every $B O$-algebra is 0 -commutative, and obtained several algebras which are logically equivalent to the $B O$-algebra. Walendziak [9] introduced a $B F$-algebra, which is a generalization of a $B$-algebra, and investigated some properties of (normal) ideals in $B F$-algebras. Kim and Kim [10] defined the notion of a $B N$-algebra, and showed that an algebra $A$ is a $B N$-algebra if and only if it is a 0-commutative $B F$-algebra. Kim et al. [11] introduced the notions of (pre-)Coxeter algebras in the Smarandache setting, and Kim and Kim [12] discussed some relations between (pre-)Coxeter algebras and its related topics.

In this paper, we introduce the notion of a $B V$-algebra consisting of 3 simple axioms, and we show it is logically equivalent to several known algebras. In particular, we investigate the role of the axiom $(B V)$ for proving the logically (non-) equivalence to several algebras. Moreover, we show that a $B V$-algebra with $(F)$ is logically equivalent to several algebras, and we show some relationships between a $B V$-algebra with $(F)$ and several related algebras.

\section{Preliminaries}

A B-algebra [6] is a non-empty set $A$ with a constant 0 and a binary operation ' $*$ ' satisfying the following axioms:

(A1) $x * x=0$,

(A2) $x * 0=x$,

(B) $(x * y) * z=x *[z *(0 * y)]$ 
for any $x, y, z \in A$

Proposition 1. [6] If $(A, *, 0)$ is a B-algebra, then $x=0 *(0 * x)$ for all $x \in A$.

An algebra $(A, *, 0)$ is called a $B H$-algebra [5] if it satisfies $(A 1),(A 2)$ and $(B H)$, where (BH) $x * y=0$ and $y * x=0$ imply $x=y$.

Kim and Kim [7] defined a BM-algebra satisfying $(A 2)$ and $(B M)$, where

(BM) $(z * x) *(z * y)=y * x$

for any $x, y, z \in A$. Walendziak [9] introduced a $B F$-algebra as an algebra $(A, *, 0)$ satisfying $(A 1),(A 2)$ and $(B F)$, where

(BF) $0 *(x * y)=y * x$

for any $x, y \in A$. An algebra $(A, *, 0)$ is called a $B O$-algebra [8] if it satisfies $(A 1),(A 2)$ and $(B O)$, where (BO) $(x * y) *(0 * z)=x *(y * z)$

for any $x, y, z \in A$. An algebra $(A, *, 0)$ is said to be 0 -commutative [13] if $x *(0 * y)=y *(0 * x)$ for any $x, y \in A$. Cho and Kim [13] discussed $B$-algebras and quasigroups using the notion of the 0-commutativity.

Proposition 2. [8] An algebra $(A, *, 0)$ is a BO-algebra if and only if it is a 0 -commutative B-algebra.

An algebra $(A, *, 0)$ is called a Coxeter algebra if it satisfies $(A 1),(A 2)$ and $(D)$, where

(D) $\quad(x * y) * z=x *(y * z)$

for all $x, y, z \in A$. It is known that a Coxeter algebra is a special type of abelian groups, i.e., a Boolean group (see [11,12]). An algebra $(A, *, 0)$ is called a pre-Coxeter algebra if it satisfies $(A 1),(A 2)$ and

(E1) if $x * y=0=y * x$, then $x=y$,

(E2) $x * y=y * x$

for all $x, y \in A$. It was proved that every Coxeter algebra is a pre-Coxeter algebra, but the converse need not be true in general (see [11,12]).

\section{3. $B V$-Algebras}

In this section, we define a notion of a $B V$-algebra, and we investigate some relations between $B V$-algebras and other algebras, i.e., $B$-algebras, 0 -commutative $B$-algebras, $B F$-algebras, $B H$-algebras, $B M$-algebras, $B N$-algebras, $B O$-algebras, $B T$-algebras and Coxeter-algebras.

Definition 1. An algebra $(A, *, 0)$ is said to be a $B V$-algebra if it satisfies $(A 1),(A 2)$ and $(B V)$, where

$(B V) \quad(x * y) * z=(0 * y) *(z * x)$

for all $x, y, z \in A$.

Example 1. Let $A:=\{0,1,2,3,4\}$ be a set with the following table:

\begin{tabular}{l|lllll}
$*$ & 0 & 1 & 2 & 3 & 4 \\
\hline 0 & 0 & 2 & 1 & 4 & 3 \\
1 & 1 & 0 & 3 & 2 & 4 \\
2 & 2 & 4 & 0 & 3 & 1 \\
3 & 3 & 1 & 4 & 0 & 2 \\
4 & 4 & 3 & 2 & 1 & 0
\end{tabular}


Then it is easy to see that $(A, *, 0)$ is a $B V$-algebra.

Proposition 3. If $(A, *, 0)$ is a $B V$-algebra, then the followings hold:

(i) $\quad x * y=(0 * y) *(0 * x)$,

(ii) $0 *(0 * x)=x$,

(iii) $x * z=0 *(z * x)$,

(iv) $(x * y) * x=0 * y$,

(v) $x *(0 * y)=y *(0 * x)$,

(vi) $(x * z) * y=(x * y) * z$,

(vii) $x * y=0 \Longrightarrow x=y$

(viii) $0 * x=0 * y \Longrightarrow x=y$,

(ix) $(x *(0 * x)) * x=x$,

(x) $\quad x * y=x * z \Longrightarrow y=z$,

(xi) $x * y=z * y \Longrightarrow x=z$,

for any $x, y, z \in A$.

Proof. (i). If we let $z:=0$ in $(B V)$, then $x * y=(x * y) * 0=(0 * y) *(0 * x)$ by (A2). (ii). If we let $y:=0$ in (i), then, by $(A 2)$ and $(A 1)$, we obtain $x=x * 0=(0 * 0) *(0 * x)=0 *(0 * x)$. (iii). If we let $y:=0$ in $(B V)$, then $(x * 0) * z=(0 * 0) *(z * x)$, and hence $x * z=0 *(z * x)$. (iv). If we let $z:=x$ in $(B V)$, then $(x * y) * x=(0 * y) *(x * x)=(0 * y) * 0=0 * y$. (v). If we let $y:=0 * y$ in (i), then $x *(0 * y)=(0 *(0 * y)) *(0 * x)$. By applying (ii), we obtain $x *(0 * y)=y *(0 * x)$. (vi). If we let $y:=z, z:=y$ in $(B V)$, then, by applying (iii) and (i), we have $(x * z) * y=(0 * z) *(y * x)=$ $(0 * z) *(0 *(x * y))=(x * y) * z$. (vii). If $x * y=0$ in (iv), then $0 * x=0 * y$, which implies $0 *(0 * x)=0 *(0 * y)$. It follows that $x=y$ by (ii). (viii). If $0 * x=0 * y$, then $0 *(0 * x)=0 *(0 * y)$ and hence $x=y$ by (ii). (ix). If we let $y:=0 * x$ in (iv), then $(x *(0 * x)) * x=0 *(0 * x)$. By applying (ii), we obtain $(x *(0 * x)) * x=x$. (x). Assume $x * y=x * z$. Then, by applying (iv), we have

$$
\begin{aligned}
0 * y & =(x * y) * x \\
& =(x * z) * x \\
& =0 * z .
\end{aligned}
$$

By applying (viii), we obtain $y=z$. (xi). If we assume $x * y=z * y$, then, by (iii), we obtain $0 *(y * x)=0 *(y * z)$. By applying (viii), we have $y * x=y * z$. By $(x)$, we obtain $x=z$.

By applying (iii) and (vii) of Proposition 3, we obtain the following theorem:

Theorem 1. Let $(A, *, 0)$ be a $B V$-algebra. Then

(i) it is a BF-algebra,

(ii) it is a BH-algebra.

Remark 1. The converse of Theorem 1(i) does not hold in general.

Example 2. Let $A:=\{0,1,2,3,4\}$ be a set. Define a binary operation "*" on $A$ as follows:

\begin{tabular}{l|lllll}
$*$ & 0 & 1 & 2 & 3 & 4 \\
\hline 0 & 0 & 1 & 2 & 4 & 3 \\
1 & 1 & 0 & 4 & 1 & 4 \\
2 & 2 & 3 & 0 & 3 & 4 \\
3 & 3 & 1 & 4 & 0 & 0 \\
4 & 4 & 3 & 3 & 0 & 0
\end{tabular}

Then $(A, *, 0)$ is a BF-algebra, but not a $B V$-algebra, since $(0 * 3) *(4 * 1)=0 \neq 4=(1 * 3) * 4$. 
Theorem 2. If $(A, *, 0)$ is a Coxeter algebra, then it is a $B V$-algebra.

Proof. Let $(A, *, 0)$ be a Coxeter algebra. Since $A$ satisfies the conditions $(A 1)$ and $(A 2)$, it is enough to show the condition $(B V)$. Given $x, y, z \in A$, we have

$$
\begin{aligned}
(0 * y) *(z * x) & =y *(z * x) \\
& =y *(x * z) \\
& =(y * x) * z \\
& =(x * y) * z
\end{aligned}
$$

Hence $A$ is a $B V$-algebra.

Remark 2. The converse of Theorem 2 does not hold in general.

Example 3. Let $(A, *, 0)$ be a $B V$-algebra as in Example 1 . Then it is not a Coxeter algebra, since $(1 * 2) * 3=$ $0 \neq 2=1 * 3=1 *(2 * 3)$.

Lemma 1. If $(A, *, 0)$ is a $B V$-algebra, then it is a 0 -commutative.

Proof. It follows immediately from Proposition 3(v).

Theorem 3. If $(A, *, 0)$ is a $B V$-algebra, then it is a B-algebra.

Proof. Let $A$ be a $B V$-algebra. Since $A$ satisfies the conditions $(A 1)$ and $(A 2)$, it is enough to show the condition $(A 3)$. If $x, y$ and $z$ be any elements of $A$, then

$$
\begin{aligned}
x *[z *(0 * y)] & =[0 *(0 * x)] *[z *(0 * y)] & & {[\because \text { Proposition 3(ii) }] } \\
& =[(0 * y) *(0 * x)] * z & & {[\because(\mathrm{BV})] } \\
& =(x * y) * z . & & {[\because \text { Proposition 3(i) }] }
\end{aligned}
$$

Hence $A$ is a $B$-algebra.

Remark 3. The converse of this theorem does not hold.

Example 4. Let $A$ be the set of all real numbers except for a negative integer $-n$. Define a binary operation "*" on $A$ as follows:

$$
x * y:=\frac{n(x-y)}{n+y}
$$

for any $x, y \in A$. Then $(A, *, 0)$ is a $B$-algebra [6], but it is not a $B V$-algebra, since $(2 * 0) * 1=\frac{n}{n+1} \neq$ $\frac{n}{2}=(0 * 0) *(1 * 2)$

By using Lemma 1 and Theorem 3, we obtain the following:

Corollary 1. If $(A, *, 0)$ is a $B V$-algebra, then it is a 0-commutative B-algebra.

Theorem 4. If $(A, *, 0)$ is a 0-commutative B-algebra, then it is a BV-algebra. 
Proof. Let $(A, *, 0)$ be a 0 -commutative $B$-algebra. Then

$$
\begin{aligned}
(0 * y) *(z * x) & =(0 * y) *[z *(0 *(0 * x))] & & {[\because \text { Proposition 1] }} \\
& =[(0 * y) *(0 * x)] * z & & {[\because(\mathrm{B})] } \\
& =(x * y) * z . & & {[\because 0 \text {-commutative }] }
\end{aligned}
$$

for all $x, y, z \in A$. Hence $(A, *, 0)$ is a $B V$-algebra.

By Corollary 1 and Theorem 4, we obtain the following corollary:

Corollary 2. An algebra $(A, *, 0)$ is a $B V$-algebra if and only if it is a 0 -commutative B-algebra.

An algebra $(A, *, 0)$ is called a BT-algebra if the conditions $(A 1),(A 2)$ and $(B T)$ hold, where

$$
[x *(0 * y)] * z=y *(z * x)
$$

for all $x, y, z \in A$

Theorem 5. An algebra $(A, *, 0)$ is a $B V$-algebra if and only if it is a BT-algebra.

Proof. Assume that $(A, *, 0)$ is a $B V$-algebra. We show the condition $(B T)$. Given $x, y, z \in A$, we have

$$
\begin{aligned}
{[x *(0 * y)] * z } & =[0 *(0 * y)] *(z * x) & & {[\because(\mathrm{BV})] } \\
& =y *(z * x) . & & {[\because \text { Proposition 3(ii) }] }
\end{aligned}
$$

for all $x, y, z \in A$. Hence $A$ is a $B T$-algebra.

Conversely, assume that $A$ is a $B T$-algebra. If we let $y=z=0$ in $(B T)$, then, by (A1) and (A2), we have $x=[x *(0 * 0)] * 0=0 *(0 * x)$ for all $x \in X$. To show that $A$ is a $B V$-algebra, we show that the condition $(B V)$ holds. In fact, we have

$$
\begin{aligned}
(0 * y) *(z * x) & =[x *(0 *(0 * y))] * z \quad[\because(\mathrm{BT})] \\
& =(x * y) * z
\end{aligned}
$$

for all $x, y, z \in A$. Hence $A$ is a $B V$-algebra.

Kim and Kim defined the notion of a $B N$-algebra. An algebra $(A, *, 0)$ is said to be a $B N$-algebra [10] if it satisfies the conditions $(A 1),(A 2)$ and $(B N)$, where

(BN) $(0 * z) *(y * x)=(x * y) * z$

for all $x, y, z \in A$. It was proved that $B N$-algebras are equivalent to 0 -commutative $B F$-algebras [10].

Theorem 6. If $(A, *, 0)$ is a $B V$-algebra, then it is a $B N$-algebra.

Proof. Let $A$ be a $B V$-algebra. Then $A$ satisfies the conditions $(A 1)$ and $(A 2)$. We show that the condition $(B N)$ holds.

$$
\begin{aligned}
(0 * z) *(y * x) & =(x * z) * y & & {[\because(\mathrm{BV})] } \\
& =(x * y) * z & & {[\because \text { Proposition 3(vi) }] }
\end{aligned}
$$

for all $x, y, z \in A$. Hence $A$ is a $B N$-algebra.

Remark 4. The converse of Theorem 6 does not hold in general. 
Example 5. Let $A:=\{0,1,2,3,4\}$ be a set with the following table:

\begin{tabular}{l|llll}
$*$ & 0 & 1 & 2 & 3 \\
\hline 0 & 0 & 1 & 2 & 3 \\
1 & 1 & 0 & 1 & 1 \\
2 & 2 & 1 & 0 & 1 \\
3 & 3 & 1 & 1 & 0
\end{tabular}

Then $(A, *, 0)$ is a BN-algebra, but not a $B V$-algebra, since $(0 * 3) *(2 * 3)=1 \neq 2=(3 * 3) * 2$.

By Corollary 2, Theorem 5 and Proposition 2, we obtain the interesting result below:

Theorem 7. The following statements are logically equivalent:

(1) $A$ is a $B V$-algebra,

(2) A is a 0-commutative B-algebra,

(3) $A$ is a BO-algebra,

(4) $A$ is a BT-algebra,

(5) $A$ is a BM-algebra.

We summarize the above discussion, and we give a diagram describing some relations between $B V$-algebras and its related algebras as follows:

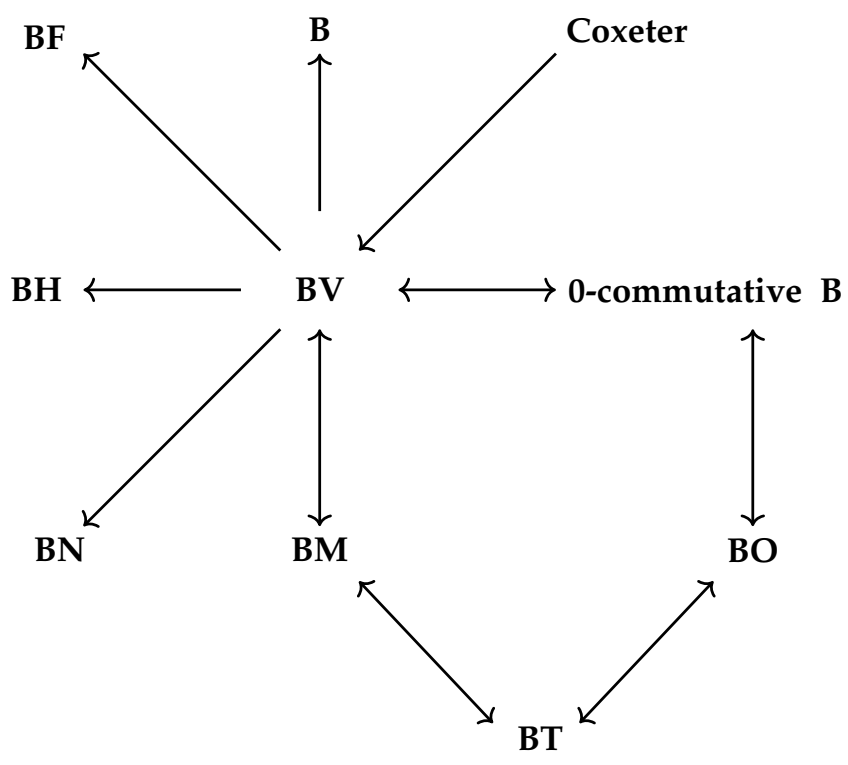




\section{BV-Algebras with Some Conditions}

In this section, we investigate some relationships between several general algebraic structures and $B V$-algebras with special conditions as follows:

(D) $\quad(x * y) * z=x *(z * y)$

(F) $\quad 0 * x=x$

for all $x, y, z \in A$. Note that Boolean groups and the Klein four group are $B V$-algebras with $(F)$.

Theorem 8. If $(A, *, 0)$ is a $B V$-algebra with $(F)$, then it is a pre-Coxeter algebra.

Proof. Let $(A, *, 0)$ be a $B V$-algebra with $(F)$. If $x * y=0=y * x$, then $x=y$ by Proposition 3(vii). On the other hand, by Proposition 3(iii), we obtain $x * y=0 *(y * x)=y * x$. Hence $A$ is a pre-Coxeter algebra.

Remark 5. The converse of Theorem 8 does not hold in general.

Example 6. Let $A:=\{0,1,2,3,4,5,6,7,8,9\}$ be a set with the following table:

\begin{tabular}{l|llllllllll}
$*$ & 0 & 1 & 2 & 3 & 4 & 5 & 6 & 7 & 8 & 9 \\
\hline 0 & 0 & 1 & 2 & 3 & 4 & 5 & 6 & 7 & 8 & 9 \\
1 & 1 & 0 & 3 & 2 & 5 & 4 & 7 & 6 & 9 & 8 \\
2 & 2 & 3 & 0 & 1 & 6 & 8 & 4 & 9 & 5 & 7 \\
3 & 3 & 2 & 1 & 0 & 9 & 7 & 8 & 5 & 6 & 4 \\
4 & 4 & 5 & 6 & 9 & 0 & 1 & 2 & 8 & 7 & 3 \\
5 & 5 & 4 & 8 & 7 & 1 & 0 & 9 & 3 & 2 & 6 \\
6 & 6 & 7 & 4 & 8 & 2 & 9 & 0 & 1 & 3 & 5 \\
7 & 7 & 6 & 9 & 5 & 8 & 3 & 1 & 0 & 4 & 2 \\
8 & 8 & 9 & 5 & 6 & 7 & 2 & 3 & 4 & 0 & 1 \\
9 & 9 & 8 & 7 & 4 & 3 & 6 & 5 & 2 & 1 & 0
\end{tabular}

Then $(A, *, 0)$ is a pre-Coxeter algebra, but it is not a $B V$-algebra with $(F)$, since $(3 * 4) * 5=6 \neq 8=$ $(0 * 4) *(5 * 3)$.

Theorem 9. An algebra $(A, *, 0)$ is a $B V$-algebra with $(F)$ if and only if it is a Coxeter algebra.

Proof. Assume that $(A, *, 0)$ is a $B V$-algebra with $(F)$. Then $A$ satisfies the conditions $(A 1)$ and $(A 2)$. Given $x, y, z \in X$, we obtain

$$
\begin{aligned}
(x * y) * z & =(0 * y) *(z * x) & & {[\because(\mathrm{BV})] } \\
& =y *(z * x) & & {[\because(\mathrm{F})] } \\
& =0 *[(z * x) * y] & & {[\because \text { Proposition 3(iii) }] } \\
& =0 *[(z * y) * x] & & {[\because \text { Proposition 3(vi) }] } \\
& =x *(y * z) . & & {[\because \text { Proposition 3(iii) }] }
\end{aligned}
$$

This shows that $A$ is a Coxeter algebra.

Conversely, assume that $(A, *, 0)$ is a Coxeter algebra. Then $A$ satisfies the conditions $(A 1)$ and $(A 2)$. Given $x, y, z \in A$, we have

$$
\begin{aligned}
(0 * y) *(z * x) & =[(0 * y) * z] * x & & {[\because \text { associativity }] } \\
& =(y * z) * x & & {[\because(\mathrm{F})] } \\
& =x *(y * z) & & {[\because \text { commutativity }] } \\
& =(x * y) * z & & {[\because \text { associativity }] }
\end{aligned}
$$


Hence $(A, *, 0)$ is a $B V$-algebra with $(F)$.

Note that the $B V$-algebra $(A, *, 0)$ in Example 1 does not satisfy the condition $(F)$, and hence it is not a Coxeter algebra.

Lemma 2. Let $(A, *, 0)$ be a $B V$-algebra. Then the followings are equivalent: for any $x, y, z \in A$,

(i) $0 * x=x$,

(ii) $\quad(x * y) * z=x *(z * y)$.

Proof. (i) $\Rightarrow$ (ii). Suppose the condition (i) holds. Then, for any $x, y, z \in A$, we have

$$
\begin{aligned}
x *(z * y) & =(0 * x) *(z * y) & & {[\because(\mathrm{i})] } \\
& =(y * x) * z & & {[\because(\mathrm{BV})] } \\
& =(0 *(x * y)) * z & & {[\because \text { Proposition 3(iii) }] } \\
& =(x * y) * z & & {[\because(\mathrm{i})] }
\end{aligned}
$$

(ii) $\Rightarrow$ (i). Suppose the condition (ii) holds. If we let $x=z=0$ in (ii), then, by (A2) and Proposition 3(ii), we obtain $0 * y=(0 * y) * 0=0 *(0 * y)=y$ for any $y \in A$.

Example 7. In Example 1, we see that $0 * 2=1 \neq 2$ and hence the condition $(x * y) * z=x *(z * y)$ does not hold for some $x, y, z \in A$. In fact, $(3 * 4) * 1=4 \neq 2=3 *(1 * 4)$.

Lemma 3. If $(A, *, 0)$ is a B-algebra with $(F)$, then $x * y=y * x$ for any $x, y \in A$.

Proof. Since $A$ is a $B$-algebra with $(F)$, if we let $x:=0$ in $(B)$, then $y * z=(0 * y) * z=0 *[z *(0 * y)]=$ $z * y$ for all $y, z \in A$.

Example 8. In Example 4, we see that $0 * y=\frac{-y}{n+y} \neq y$ and hence the condition $x * y=y * x$ does not hold for some $x, y \in A$. In fact, $3 * 4=\frac{-n}{n+4} \neq \frac{n}{n+3}=4 * 3$.

Theorem 10. An algebra $(A, *, 0)$ is a BV-algebra with $(F)$ if and only if it is a B-algebra with $(F)$.

Proof. Let $(A, *, 0)$ be a $B V$-algebra with the condition $(F)$. Then, for any $x, y, z \in A$, we have

$$
\begin{aligned}
x *[z *(0 * y)] & =x *(z * y) & & {[\because(\mathrm{F})] } \\
& =(y * z) * x & & {[\because \text { Lemma } 2] }
\end{aligned}
$$

Hence $(A, *, 0)$ is a $B$-algebra with $(F)$.

Conversely, let $A$ be a $B$-algebra with $(F)$. Then

$$
\begin{aligned}
(0 * y) *(z * x) & =y *[z *(0 * x)] & & {[\because(\mathrm{F})] } \\
& =(y * x) * z & & {[\because(\mathrm{B})] } \\
& =(x * y) * z & & {[\because \text { Lemma 3] }}
\end{aligned}
$$

for any $x, y, z \in A$. Hence $A$ is a $B V$-algebra with $(F)$.

Lemma 4. If $(A, *, 0)$ is a $B V$-algebra with $(F)$, then $x * y=y * x$ for any $x, y \in A$.

Proof. Since $(A, *, 0)$ is a $B V$-algebra with $(F)$, by Proposition 3(iii) and $(F)$, we have $x * y=0 *(y *$ $x)=y * x$ for any $x, y \in A$. 
Lemma 5. If $(A, *, 0)$ is a BF-algebra with $(D)$, then

(i) $0 * x=x$,

(ii) $x * y=y * x$.

for any $x, y \in A$.

Proof. (i). Let $A$ be a $B F$-algebra with $(D)$. If we let $y:=x, z:=x$ in $(D)$, then $(x * x) * x=x *(x * x)$ and hence $0 * x=x * 0=x$ for all $x \in A$.

(ii). If we let $x:=0$ in $(D)$, then $y * z=(0 * y) * z=0 *(z * y)=z * y$ by (i) for any $y, z \in A$.

Theorem 11. An algebra $(A, *, 0)$ is a BV-algebra with $(F)$ if and only if it is a BF-algebra with $(D)$.

Proof. Assume that $A$ is a $B V$-algebra with $(F)$. Then $A$ satisfies the conditions (A1) and (A2). By Proposition 3(iii), $A$ satisfies the condition $(B F)$. Let $x, y$ and $z$ be any elements of $A$. Then

$$
\begin{aligned}
(x * y) * z & =(y * x) * z & & {[\because \text { Lemma } 4] } \\
& =(0 * x) *(z * y) & & {[\because(\mathrm{BV})] } \\
& =x *(z * y) . & & {[\because(\mathrm{F})] }
\end{aligned}
$$

Hence $A$ is a $B F$-algebra with $(D)$.

Conversely, assume that $A$ is a $B F$-algebra with $(D)$. Then $A$ satisfies the conditions (A1) and (A2). By Lemma 5(i), $A$ satisfies the condition $(F)$. Let $x, y$ and $z$ be any elements of $A$. Then

$$
\begin{aligned}
(0 * y) *(z * x) & =y *(z * x) & & {[\because(\mathrm{F})] } \\
& =(y * x) * z & & {[\because(\mathrm{D})] } \\
& =(x * y) * z . & & {[\because \text { Lemma } 5(\mathrm{ii})] }
\end{aligned}
$$

Hence $A$ is a $B V$-algebra with $(F)$.

Lemma 6. Let $(A, *, 0)$ be a BN-algebra with $(D)$. Then

(i) $0 *(x * y)=y * x$,

(ii) $0 *(0 * x)=x$,

(iii) $0 * x=x$,

(iv) $x * y=y * x$,

for any $x, y \in A$.

Proof. Let $A$ be a $B N$-algebra with $(D)$. (i). If we let $z:=0$ in $(B N)$, then $(0 * 0) *(y * x)=(x * y) * 0$. By applying $(A 1)$ and $(A 2)$, we obtain $0 *(y * x)=x * y$.

(ii). If we let $y:=0, z:=0$ in $(B N)$, then $(0 * 0) *(0 * x)=(x * 0) * 0$. By applying $(A 1)$ and $(A 2)$, we obtain $0 *(0 * x)=x$.

(iii). Let $x:=0, z:=0$ in $(D)$. Then $(0 * y) * 0=0 *(0 * y)$. By applying (ii) and (A2), we obtain $y=0 *(0 * y)=(0 * y) * 0=0 * y$ for all $y \in A$.

(iv). If we let $x:=0$ in $(B N)$, then $(0 * z) *(y * 0)=(0 * y) * z$. By applying (iii) and $(A 2)$, we obtain $z * y=y * z$ for all $y, z \in A$.

Theorem 12. An algebra $(A, *, 0)$ is a $B V$-algebra with $(F)$ if and only if it is a BN-algebra with $(D)$.

Proof. Let $(A, *, 0)$ be a $B V$-algebra with $(F)$. Then $A$ satisfies the conditions (A1) and (A2). By Lemma 2, $A$ satisfies the condition $(D)$. By applying Proposition 3(vi), we obtain $(0 * z) *(y * x)=$ 
$(x * z) * y=(x * y) * z$ for all $x, y, z \in A$, which shows that the condition $(B N)$ holds. Hence $(A, *, 0)$ is a $B N$-algebra with $(D)$.

Conversely, let $A$ be a $B N$-algebra with $(D)$. Then $A$ satisfies the condition (A1) and (A2). By Lemma 6(iii), $A$ satisfies the condition $(F)$. Given $x, y, z \in A$, we have

$$
\begin{aligned}
(0 * y) *(z * x) & =y *(z * x) & & {[\because \text { Lemma 6(iii) }] } \\
& =(y * x) * z & & {[\because(\mathrm{D})] } \\
& =(x * y) * z . & & {[\because \text { Lemma 6(iv) }] }
\end{aligned}
$$

Hence $A$ is a $B V$-algebra with $(F)$.

From Lemma 2, we have the following corollary:

Corollary 3. An algebra $(A, *, 0)$ is a $B V$-algebra with $(F)$ if and only if it is a $B V$-algebra with $(D)$.

Theorem 13. Let $(A, *, 0)$ be a B-algebra. Then the followings are equivalent: for any $x, y, z \in A$,

(i) $0 * x=x$,

(ii) $\quad(x * y) * z=x *(z * y)$.

Proof. (i) $\Rightarrow$ (ii). Let $A$ be a $B$-algebra. Assume $A$ satisfies the condition (i). Then

$$
\begin{aligned}
(x * y) * z & =x *[z *(0 * y)] & & {[\because(\mathrm{B})] } \\
& =x *(z * y) & & {[\because(\mathrm{i})] }
\end{aligned}
$$

for any $x, y, z \in A$.

(ii) $\Rightarrow$ (i). Assume $A$ satisfies the condition (ii). Then

$$
\begin{aligned}
x & =0 *(0 * x) & & {[\because \text { Proposition } 1] } \\
& =(0 * x) * 0 & & {[\because(\mathrm{ii})] } \\
& =0 * x & & {[\because(\mathrm{A} 2)] }
\end{aligned}
$$

for any $x \in A$.

Lemma 7. Let $(A, *, 0)$ be a BO-algebra with $(F)$. Then, for any $x, y, z \in A$, we have

(i) $(x * y) * z=x *(y * z)$,

(ii) $\quad x * y=y * x$.

Proof. (i). Since $A$ is a $B O$-algebra with $(F)$, we have

$$
\begin{aligned}
(x * y) * z & =(x * y) *(0 * z) & & {[\because(\mathrm{F})] } \\
& =x *(y * z) & & {[\because(\mathrm{BO})] }
\end{aligned}
$$

for any $x, y, z \in A$.

(ii). By (i), $(A, *, 0)$ is a Coxeter algebra, and hence $x * y=y * x$ for all $x, y \in A$.

By Theorem 7, we know that a $B V$-algebra is logically equivalent to a $B O$-algebra, but there is no direct proof of its equivalence. Using the condition $(F)$, we describe its relationship as below:

Theorem 14. An algebra $(A, *, 0)$ is a $B V$-algebra with $(F)$ if and only if it is a BO-algebra with $(F)$. 
Proof. Let $(A, *, 0)$ be a $B V$-algebra with $(F)$. We show that the condition $(B)$ holds. If we take $x, y$ and $z$ in $A$, then

$$
\begin{aligned}
(x * y) *(0 * z) & =(x * y) * z & & {[\because(\mathrm{F})] } \\
& =z *(y * x) & & {[\because \text { Theorem } 8] } \\
& =(0 * z) *(y * x) & & {[\because(\mathrm{F})] } \\
& =(x * z) * y & & {[\because(\mathrm{BV})] } \\
& =y *(x * z) & & {[\because \text { Theorem } 8] } \\
& =(0 * y) *(x * z) & & {[\because(\mathrm{F})] } \\
& =(z * y) * x & & {[\because(\mathrm{BV})] } \\
& =x *(y * z) . & & {[\because \text { Theorem } 8] }
\end{aligned}
$$

Hence $A$ is a $B O$-algebra with $(F)$.

Conversely, assume that $A$ is a $B O$-algebra with $(F)$. We show the condition $(B V)$. Given $x, y, z \in A$, by using Lemma $7(i)$ and $(F)$, we obtain:

$$
\begin{aligned}
(0 * y) *(z * x) & =y *(z * x) \\
& =(y * z) * x \\
& =x *(y * z) \\
& =(x * y) * z
\end{aligned}
$$

Hence $A$ is a $B V$-algebra with $(F)$.

Theorem 15. If $(A, *, 0)$ is a $B V$-algebra with $(F)$, then it is a BN-algebra with $(F)$.

Proof. Assume that $(A, *, 0)$ is a $B V$-algebra with $(F)$. We prove the condition $(B N)$. Given $x, y, z \in A$, we have

$$
\begin{aligned}
(0 * z) *(y * x) & =(x * z) * y & & {[\because(\mathrm{BV})] } \\
& =y *(z * x) & & {[\because \text { Theorem } 8] } \\
& =(0 * y) *(z * x) & & {[\because(\mathrm{F})] } \\
& =(x * y) * z . & & {[\because(\mathrm{BV})] }
\end{aligned}
$$

Hence $A$ is a $B N$-algebra with $(F)$.

Remark 6. The converse of Theorem 15 does not hold in general.

Example 9. Let $A:=\{0,1,2\}$ be a set with the following table:

\begin{tabular}{l|lll}
$*$ & 0 & 1 & 2 \\
\hline 0 & 0 & 1 & 2 \\
1 & 1 & 0 & 1 \\
2 & 2 & 1 & 0
\end{tabular}

Then it is easy to see that $A$ is a BN-algebra with $(F)$, but not a $B V$-algebra with $(F)$, since $(1 * 2) * 1=$ $1 * 1=0 \neq 2=2 * 0=(0 * 2) *(1 * 1)$.

From the above theorems, we obtain the following equivalent statements: 
Corollary 4. The followings are equivalent:

(i) $(A, *, 0)$ is a $B V$-algebra with $(F)$,

(ii) $(A, *, 0)$ is a B-algebra with $(F)$,

(iii) $(A, *, 0)$ is a BO-algebra with $(F)$,

(iv) $(A, *, 0)$ is a BF-algebra with $(D)$,

(v) $(A, *, 0)$ is a $B N$-algebra with $(D)$,

(vi) $(A, *, 0)$ is a $B V$-algebra with $(D)$,

(vii) $(A, *, 0)$ is a Coxeter algebra,

(viii) $(A, *, 0)$ is a Boolean group.

We provide a diagram describing some relations between $B V$-algebras with $(F)$ and its related algebras as follows:

BN \& (D)

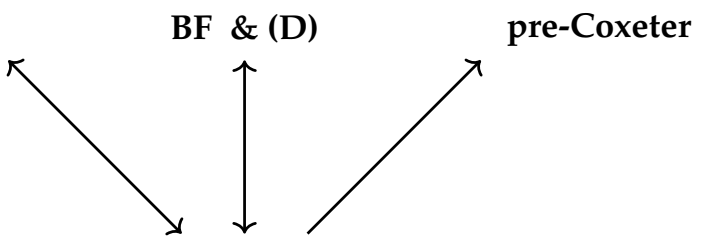

BV \& (D) $\longleftrightarrow$ BV \& (F) $\longleftrightarrow$ BO \& (F)

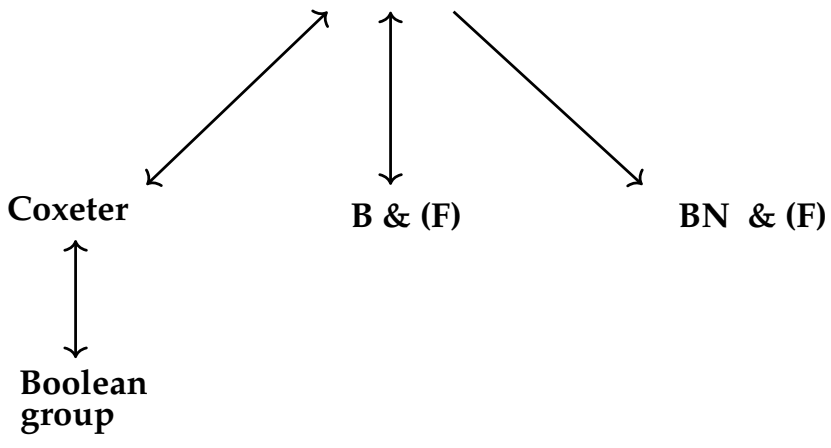

\section{Conclusions and Future Works}

We introduced the notion of a $B V$-algebra, and investigated some relations between $B V$-algebras and their related topics. Moreover, by using some additional axioms, we discussed some relations between $B V$-algebras with $(F)$ and several algebraic structures with additional axioms. In particular, we have focussed on the axiom $(B V)$, and investigated some relations with several other axioms which are discussed in various different algebraic structures. We found that the role of the axiom $(B V)$ is important in studying general algebraic structures.

We will discuss several ideal theories, isomorphism theorems in $B V$-algebras, and some applications to fuzzy theory, soft set theory and neutrosophic theory in the sequel. Moreover, we will investigate the axiom (BT) for further development of general algebraic structures.

Author Contributions: Conceptualization and Supervision: I.H.H.; Investigation: H.S.K.; Resources: Y.L.L. All authors have read and agreed to the published version of the manuscript.

Funding: This research received no external funding.

Acknowledgments: The authors are deeply grateful to Chang Bum Kim for his valuable suggestions and help. The authors would like to express their great thanks for referee's valuable suggestions and help.

Conflicts of Interest: The author declares no conflict of interest. 


\section{References}

1. Iséki, K.; Tanaka, S. An introduction to the theory of BCK-algebras. Math. Jpn. 1978, 23, 1-26.

2. Huang, Y.S. BCI-Algebras; Science Press: Beijing, China, 2006.

3. Hu, Q.P.; Li, X. On BCH-algebras. Kobe J. Math. 1983, 11, 313-320.

4. Dudek, W.A. A new characterization of ideals in BCC-algebras. Novi Sad J. Math. 1999, 29, 139-145.

5. Jun, Y.B.; Roh, E.H.; Kim, H.S. On BH-algebras. Sci. Math. Jpn. 1998, 1, 347-354.

6. Neggers, J.; Kim, H.S. On B-algebras. Math. Vesnik. 2002, 54, 21-29.

7. Kim, C.B.; Kim, H.S. On BM-algebra. Sci. Math. Jpn. 2006, 63, 421-427.

8. Kim, C.B.; Kim, H.S. On BO-algebra. Math. Slovaca 2012, 62, 421-427. [CrossRef]

9. Walendcziak, A. On BF-algebras. Math. Slovaca 2007, 57, 119-128. [CrossRef]

10. Kim, C.B.; Kim, H.S. On BN-algebra. Kyungpook Math. J. 2013, 53, 175-184. [CrossRef]

11. Kim, H.S.; Kim, Y.H.; Neggers, J. Coxeters and pre-Coxeter algebras in Smarandache setting. Honam Math. J. 2004, 26, 471-481.

12. Kim C.B.; Kim, H.S. (Pre-)Coxeter algebras and related topics. Math. Slovaca 2010, 60, 33-42. [CrossRef]

13. Cho, J.R.; Kim, H.S. On B-algebras and quasigroups. Quasigroups Relat. Syst. 2001, 8, 1-6.

Publisher's Note: MDPI stays neutral with regard to jurisdictional claims in published maps and institutional affiliations.

(C) 2020 by the authors. Licensee MDPI, Basel, Switzerland. This article is an open access article distributed under the terms and conditions of the Creative Commons Attribution (CC BY) license (http://creativecommons.org/licenses/by/4.0/). 